\title{
Geometrical Correlation between Extra-dimensions and Universe Accelerated Expansion
}

\author{
H. Ruchvarger \\ Department of Mathematics, Sami Shamoon College of Engineering, Israel
}

$\&$

Faculty of Natural Science, Ben-Gurion University, Israel

Received: August 26, $2011 \quad$ Accepted: September 13, $2011 \quad$ Published: November 1, 2011
doi:10.5539/apr.v3n2p110
URL: http://dx.doi.org/10.5539/apr.v3n2p110

\begin{abstract}
Multidimensional space-times are represented as curved surfaces embedded in higher dimensional flat spaces. The embedding of each surface is based on geometrical principles. According to these geometrical principles, we use variable separated coordinates so that the coordinates parameters become an orthogonal curved coordinates system for each space-time surface. In this way, we obtain that the universe expands and that the expansion is accelerated. By using co-moving coordinates and assuming that there is at least one geodesic which represent a straight line in the curved multidimensional space-time surface (this is a kind of "equivalence principle" of a new type), we obtain the curved multidimensional space-time surface's equation, its metric and accelerated expanded three-sphere surface's particles that also explains the accelerated expansion of the universe.
\end{abstract}

Keywords: Geometrical principles, Multidimensional space-time, Accelerated expansion Universe

\section{Introduction}

The study of multidimensional space-times started with the Kaluza and Klein (T.Appelquist, et al.,1987) (Paul K. Townsend and N.R. Wohlfarth,2003), who studied 5D space-times when the extra-dimensions are compact.

In recent years more general types of space-times have been considered, like multidimensional space-times which are De-Sitter multidimensional-sphere surfaces considering the energy density of the vacuum (Y.B. Zeldovich, 1967) (Paul K. Townsend and Mattias N.R.Wohlfarth,2003) (Mauricio Bellini, 2006).

Also, 5D curved space-time surfaces with a finite and an infinite extra-dimensions was studied (Y. Kim,et al., 2002) (E.A. Bergshoff, et al., 2005) and 5D flat space-times with an infinite extra-dimension, based on De-Sitter metric considering the energy density of the vacuum, as Wesson,s model (P.S. Wesson, 1999) (Tomas Liko and Paul S. Wesson, 2005).

Other explanations to the universe accelerated expansion are based on interacting a scalar field with non-negative potential often referred to as "dark energy" (E.I. Guendelman and A.B. Kaganovich, 2004). different 5D space-time model is Carmeli's accelerating universe model (S. Behar and M. Carmeli, 2000) (M. Carmeli, 2002) (J. Hartnett, 2005) in which the extra-dimension is the radial velocity of the galaxies in the expanding universe.

A five dimensional FLRW type Kaluza-Klein cosmological model with static extra-dimension has been studied in Ref. (K.Purohit, Bhatt and Yogesh, 2011).An inflationary model in 6D super-gravity has been constructed in Ref. (L.V. Nirop and C.P. Burgess, 2011), based on explicit time-depended solutions to the full higher-dimensional field equation, back-reacting to the presence of a 4D inflation rolling on a space-filling co-dimension-2 source brane.

In our opinion, the best explanation for something that is unknown, is by using extra-dimensions. Therefore, we explained the universe accelerated expansion by using extra-dimensions (E. Guendelman and H. Ruchvarger, 2004).

In (E. Guendelman and H. Ruchvarger, 2004), we explain the universe accelerated expansion by defining a general orthogonal multidimensional variable separated coordinate system based on a three-sphere surface.

In (E. Guendelman and H. Ruchvarger, 2004), we find the extra-dimensional metric component by a new equivalence principle that is an additional principle to Einstein's equivalence principle. 
But by that new equivalence principle, we find only the first extra-dimensional metric component and to explain the universe acceleration expansion by an orthogonal multidimensional variable separated coordinate system, we need at least two extra-dimensions.

In this article, we find the orthogonal multidimensional variable separated coordinate system that represents the embedded curved multidimensional space-time surface,

by a special form of equations for the coordinates, based on geometrical principles.

By this orthogonal multidimensional variable separated coordinate system, we obtain that the universe expands and that the expansion is accelerated, without the need of considering the energy density of the vacuum as in other studies (Y.B. Zeldovich,1967) (Paul K. Townsend and Mattias N.R.Wohlfarth,2003) (Mauricio Bellini, 2006) and (P.S. Wesson, 1999) (Tomas Liko and Paul S. Wesson, 2005) .

By this orthogonal curved multidimensional space-time surface coordinates, we also obtain the three-sphere surface coordinates, the metric components of all dimensions, including the extra-dimensions and the curved multidimensional space-time surface equation, for all the extra-dimensions that we want to use.

\section{Geometrical principles for the curved multidimensional space-time construction}

The multidimensional space-time is represented as a curved surface embedded in a flat space

$$
d S_{n+1}^{2}=-d X_{t}^{2}+\sum_{i=1}^{4} d X_{i}^{2}+\sum_{j=5}^{n} \pm d X_{j}^{2}
$$

and defined by an orthogonal variable separated coordinate system.

Each additional coordinate after the first two coordinates $X_{1}$ and $X_{2}$, depends only on an additional parameter while the former coordinates depend on the former parameters and on the additional parameters too, according to the following form

$$
X_{2}=\prod_{m=1}^{n} p_{2 m}\left(f_{m}\right) \quad, \quad X_{k}=\prod_{m=k}^{n} p_{k m}\left(f_{m}\right) \quad, \quad k=1,3,4, t, 5,6, \ldots, n
$$

The form (2) is explained by the following geometrical principles:

The coordinates $X_{1}, X_{2}, X_{3}, X_{4}$ are the embedding of the curved 3D surface in the curved 4D space-time surface, the coordinate $X_{t}$ is a function of time and the coordinates $X_{5}, \ldots X_{n}$ represent the extra-dimensions.

The two horizontal axes $X_{1}$ and $X_{2}$ represent horizontal planes in the multidimensional hyper-plane. Therefore, the coordinates $X_{1}$ and $X_{2}$ depend on an horizontal angle $\varphi$.

The axis $X_{3}$ does not depend on these horizontal planes and therefore does not depend on the horizontal angle $\varphi$, but depends on a new parameter that is the vertical angle $\theta$. The axes $X_{1}, X_{2}$ and $X_{3}$ represent 3D hyper-planes.

The axis $X_{4}$ does not depend on these 3D hyper-planes and therefore does not depend on the angles $\varphi$ and $\theta$ in these 3D hyper-planes, but depends on a new angle $\chi$ that is perpendicular to the angles $\varphi$ and $\theta$. The axes $X_{1}, X_{2}, X_{3}$ and $X_{4}$ represent $4 \mathrm{D}$ hyper-planes including the curved $3 \mathrm{D}$ surfaces represented by the angles $\varphi$, $\theta$ and $\chi$.

The axis $X_{t}$ does not depend on these 4D hyper-planes and therefore does not depend on the angles $\varphi, \theta$ and $\chi$ in these 4D hyper-planes, but depends on the time $t$ that is a new parameter. The time $t$ is orthogonal to the curved 3D surfaces that are represented by the angles $\varphi, \theta$ and $\chi$. Thus, $t$ is orthogonal to $\varphi, \theta$ and $\chi$.The axes $X_{1}, X_{2}, X_{3}, X_{4}$ and $X_{t}$ represent 5D hyper-planes including the curved 4D space-time surfaces represented by the angles $\varphi, \theta, \chi$ and to the time $t$.

The extra-axis $X_{5}$ does not depend on these 5D hyper-planes and therefore does not depend on the angles $\varphi, \theta, \chi$ and on the time $t$ that are the curved 4D space-time surfaces parameters, but depends on a new parameter $f_{5}$. The parameter $f_{5}$ is orthogonal to the curved 4D space-times that are represented by the angles $\varphi, \theta, \chi$ and the time $t$.

Thus, $f_{5}$ is orthogonal to $\varphi, \theta, \chi$ and to $t$. The axes $X_{1}, X_{2}, X_{3}, X_{4}, X_{t}, X_{5}$ represent $6 \mathrm{D}$ hyper-planes including the curved $5 \mathrm{D}$ space-time surfaces represented by the parameters $\varphi, \theta, \chi, t, f_{5}$ and so on for the 
additional extra-dimensions.

Thus, the parameters of the coordinates are the curved orthogonal coordinates of each multidimensional space-time surface, means that the metric of each space-time surface will not have off-diagonal components (Appendix A).

\section{The multidimensional coordinate system according to the geometrical principles}

According to the geometrical principles represented by (2), we define the curved multidimensional surface's coordinate system as follows

$$
\begin{aligned}
& X_{1}=\prod_{m=1}^{n} p_{1 m}\left(f_{m}\right)=p_{1 t}(t) \cdot p_{1 \chi}(\chi) \cdot p_{1 \theta}(\theta) \cdot p_{1 \varphi}(\varphi) \cdot \prod_{m=5}^{n} p_{1 m}\left(f_{m}\right), \\
& X_{2}=\prod_{m=1}^{n} p_{2 m}\left(f_{m}\right)=p_{2 t}(t) \cdot p_{2 \chi}(\chi) \cdot p_{2 \theta}(\theta) \cdot p_{2 \varphi}(\varphi) \cdot \prod_{m=5}^{n} p_{2 m}\left(f_{m}\right), \\
& X_{3}=\prod_{m=2}^{n} p_{3 m}\left(f_{m}\right)=p_{3 t}(t) \cdot p_{3 \chi}(\chi) \cdot p_{3 \theta}(\theta) \cdot \prod_{m=5}^{n} p_{3 m}(f), \\
& X_{4}=\prod_{m=3}^{n} p_{4 m}\left(f_{m}\right)=p_{4 t}(t) \cdot p_{4 \chi}(\chi) \cdot \prod_{m=5}^{n} p_{4 m}(f), \\
& X_{t}=\prod_{m=4}^{n} p_{t m}\left(f_{m}\right)=p_{t t}(t) \cdot \prod_{m=5}^{n} p_{t m}\left(f_{m}\right)
\end{aligned}
$$

and

$$
X_{j}=\prod_{m=j}^{n} p_{j m}\left(f_{m}\right)
$$

where $\varphi \equiv f_{1} \quad, \quad \theta \equiv f_{2} \quad, \quad \chi \equiv f_{3} \quad, \quad t \equiv f_{4}$ and $j \geq 5$.

According to the geometrical principles that we assume, the coordinates parameters represent a curved orthogonal coordinate system for the curved multidimensional space-time surface. Thus, the curved multidimensional metric will not have off-diagonal components, which means that

$$
g_{\varphi f_{m}}=g_{\varphi t}=g_{\varphi \chi}=g_{\varphi \theta}=g_{\theta f_{m}}=g_{\theta t}=g_{\theta \chi}=g_{f_{m} \chi}=g_{t \chi}=0
$$

for each $m \geq 5$.

By the variable separate coordinates (3) and by (5), we obtain the following equations

$$
\begin{aligned}
& p_{2 \theta}=p_{1 \theta} \equiv p_{\theta} \quad, \quad p_{3 \chi}=p_{2 \chi}=p_{1 \chi} \equiv p_{\chi} \quad, \quad p_{4 t}=p_{3 t}=p_{2 t}=p_{1 t} \equiv p_{t}, \\
& \prod_{m=5}^{n} p_{4 m}^{2}=\prod_{m=5}^{n} p_{3 m}^{2}=\prod_{m=5}^{n} p_{2 m}^{2}=\prod_{m=5}^{n} p_{1 m}^{2} \equiv \prod_{m=5}^{n} p_{m}^{2}, \\
& -p_{2 \varphi}^{2}=p_{1 \varphi}^{2}-1 \quad, \quad-p_{3 \theta}^{2}=p_{\theta}^{2}-1 \quad, \quad-p_{4 \chi}^{2}=p_{\chi}^{2}-1
\end{aligned}
$$

(Appendix B) in which each curved 3D space surface becomes a three-sphere surface

$$
\sum_{i=1}^{4} X_{i}^{2}=p_{t}^{2}\left(\frac{t}{K_{t}}\right) \cdot \prod_{j=5}^{n} p_{j}^{2}\left(f_{j}\right)
$$

with the radius

$$
\sqrt{\sum_{i=1}^{4} X_{i}^{2}}=p_{t}(t) \cdot \prod_{j=5}^{n} p_{j}\left(f_{j}\right)
$$

and the following coordinates 


$$
\begin{aligned}
& X_{1}=p_{t}(t) \cdot \sin (\chi) \cdot \sin (\theta) \cdot \cos (\varphi) \cdot \prod_{m=5}^{n} p_{m}\left(f_{m}\right)=p_{t}(t) \cdot x_{1} \cdot \prod_{m=5}^{n} p_{m}\left(f_{m}\right), \\
& X_{2}=p_{t}(t) \cdot \sin (\chi) \cdot \sin (\theta) \cdot \sin (\varphi) \cdot \prod_{m=5}^{n} p_{m}\left(f_{m}\right)=p_{t}(t) \cdot x_{2} \cdot \prod_{m=5}^{n} p_{m}\left(f_{m}\right), \\
& X_{3}=p_{t}(t) \cdot \sin (\chi) \cdot \cos (\theta) \cdot \prod_{m=5}^{n} p_{m}\left(f_{m}\right)=p_{t}(t) \cdot x_{3} \cdot \prod_{m=5}^{n} p_{m}\left(f_{m}\right), \\
& X_{4}=p_{t}(t) \cdot \cos (\chi) \cdot \prod_{m=5}^{n} p_{m}\left(f_{m}\right)=p_{t}(t) \cdot x_{4} \cdot \prod_{m=5}^{n} p_{m}\left(f_{m}\right)
\end{aligned}
$$

in which: $0 \leq \varphi \leq 2 \pi, 0 \leq \theta \leq \pi$ and $0 \leq \chi \leq \pi \quad$ (Fig.1).

\section{The explanation for the universe accelerated expansion}

According to the coordinates parameters that represent a curved orthogonal coordinate system for the curved multidimensional space-time surface, we obtain

$$
g_{t f_{m}}=0=2 \sum_{i=1}^{4} \frac{\partial X_{i}}{\partial f_{m}} \cdot \frac{\partial X_{i}}{\partial t}-2 \frac{\partial X_{t}}{\partial f_{m}} \cdot \frac{\partial X_{t}}{\partial t}
$$

for each $m \geq 5$

By (3),(6) and (10), we obtain for each $m \geq 5$, the following equations

$$
p_{t t}^{2}=C_{t} \cdot p_{t}^{2}-B_{t} \quad, \quad C_{t} \cdot \prod_{m=5}^{n} p_{t m}^{2}=\prod_{m=5}^{n} p_{m}^{2}
$$

and the coordinate

$$
X_{t}=p_{t t} \cdot \prod_{m=5}^{n} p_{t m}=\sqrt{p_{t}^{2}-K_{t}} \cdot \prod_{m=5}^{n} p_{m}
$$

where $B_{t}=$ const.,$C_{t}=$ const. and $K_{t} \equiv \frac{B_{t}}{C_{t}}$ (Appendix C).

Thus, by (3),(6) and (11), time's metric component becomes as follows

$$
\begin{aligned}
& g_{t t}=\sum_{i=1}^{4}\left(\frac{\partial X_{i}}{\partial t}\right)^{2}-\left(\frac{\partial X_{t}}{\partial t}\right)^{2}=p_{\chi}^{2} \cdot p_{\theta}^{2} \cdot p_{1 \varphi}^{2} \cdot\left(\frac{d p_{t}}{d t}\right)^{2} \cdot \prod_{m=5}^{n} p_{m}^{2}+ \\
& +p_{\chi}^{2} \cdot p_{\theta}^{2} \cdot p_{2 \varphi}^{2} \cdot\left(\frac{d p_{t}}{d t}\right)^{2} \cdot \prod_{m=5}^{n} p_{m}^{2}+p_{\chi}^{2} \cdot p_{3 \theta}^{2} \cdot\left(\frac{d p_{t}}{d t}\right)^{2} \cdot \prod_{m=5}^{n} p_{m}^{2}+ \\
& +p_{4 \chi}^{2} \cdot\left(\frac{d p_{t}}{d t}\right)^{2} \cdot \prod_{m=5}^{n} p_{m}^{2}-\left(\frac{d p_{t t}}{d t}\right)^{2} \cdot \prod_{m=5}^{n} p_{t m}^{2}=\left[\left(\frac{d p_{t}}{d t}\right)^{2}-\frac{1}{C_{t}} \cdot\left(\frac{d p_{t t}}{d t}\right)^{2}\right] \cdot \prod_{m=5}^{n} p_{m}^{2}= \\
& =\left(\frac{d p_{t}}{d t}\right)^{2} \cdot\left[1-\frac{C_{t} \cdot p_{t}^{2}}{C_{t} \cdot p_{t}^{2}-B_{t}}\right] \cdot \prod_{m=5}^{n} p_{m}^{2}=\frac{-K_{t}}{p_{t}^{2}-K_{t}} \cdot\left(\frac{d p_{t}}{d t}\right)^{2} \cdot \prod_{m=5}^{n} p_{m}^{2}\left(f_{m}\right)
\end{aligned}
$$

in which

$$
g_{t t} \cdot d t^{2}=\frac{-K_{t} \cdot d p_{t}^{2}}{p_{t}^{2}-K_{t}} \cdot \prod_{j=5}^{n} p_{j}^{2}\left(f_{j}\right)
$$

By defining $\frac{K_{t} \cdot d p_{t}^{\prime 2}}{p_{t}^{\prime 2}-K_{t}}=d T^{2} \quad, \quad p_{t}^{\prime}=\sqrt{K_{t}} \cosh (x)$ and integration, we obtain 


$$
K_{t} \int_{\sqrt{K_{t}}}^{p_{t}} \frac{d p_{t}^{\prime}}{\sqrt{p_{t}^{\prime 2}-K_{t}}}=K_{t} \int_{0}^{\cosh ^{-1}\left(\frac{p_{t}}{\sqrt{K_{t}}}\right)} d x=K_{t} \cdot \cosh ^{-1}\left(\frac{p_{t}}{\sqrt{K_{t}}}\right)=\int_{0}^{t} d T=t
$$

Thus,

$$
p_{t}=\sqrt{K_{t}} \cdot \cosh \left(\frac{t}{K_{t}}\right), K_{t}=\frac{1}{H_{0}}
$$

By substituting (16) in (8), each three-sphere surface's radius, becomes

$$
R_{3}=\sqrt{\sum_{i=1}^{4} X_{i}^{2}}=\sqrt{K_{t}} \cdot \cosh \left(\frac{t}{K_{t}}\right) \cdot \prod_{j=5}^{n} p_{j}\left(f_{j}\right)
$$

that represents for each constant value of $\prod_{j=5}^{n} p_{j}\left(f_{j}\right)$, a three-sphere subspace's accelerated expansion. By substituting (16), (17) in (12), we obtain for each constant value of $\prod_{m=5}^{n} p_{m}^{2}$, a four-Dimensional space-time universe embedding infinite three-sphere surfaces represented by (18) (Fig.2):

$$
\begin{aligned}
& X_{t}=\sqrt{K_{t} \cdot \cosh ^{2}\left(\frac{t}{K_{t}}\right)-K_{t} \cdot \prod_{m=5}^{n} p_{m}, X_{t}^{2}=K_{t} \cdot \cosh ^{2}\left(\frac{t}{K_{t}}\right) \cdot \prod_{m=5}^{n} p_{m}^{2}-K_{t} \cdot \prod_{m=5}^{n} p_{m}^{2}=} \\
& =\sum_{i=1}^{4} X_{i}^{2}-K_{t} \cdot \prod_{m=5}^{n} p_{m}^{2}, \quad \sum_{i=1}^{4} X_{i}^{2}-X_{t}^{2}=K_{t} \cdot \prod_{m=5}^{n} p_{m}^{2}
\end{aligned}
$$

Thus, by a special form of the equations (3) and (4) for the curved orthogonal multidimensional surface coordinates, based on (2), we show the universe accelerated expansion (17) without the need of considering the energy density of the vacuum.

Also, in order to obtain the universe accelerated expansion, we have to use at least two extra-dimensions in which the curved multidimensional space-time surface is at least

a curved 6D space-time surface.

\section{Extra-coordinates, extra-metric-components and curved multidimensional surface equations}

For more than 6-dimensional space-time surface, according to the curved orthogonal coordinate system we obtain that $g_{f_{5} f_{j}}=0$, for each $j \geq 6$.

By (4),(6),(12) and (16) we obtain the first extra-coordinate

$$
X_{5}=\sqrt{\mp K_{t} \cdot\left[p_{5}^{2}\left(f_{5}\right)-K_{5}\right]} \cdot \prod_{j=6}^{n} p_{j}\left(f_{j}\right)
$$

(Appendix D) and

$$
g_{f_{5} f_{5}} \cdot d f_{5}^{2}=\sum_{i=1}^{5}\left(\frac{\partial X_{i}}{\partial f_{5}}\right)^{2} d f_{5}^{2}=\frac{K_{t} \cdot K_{5} \cdot \prod_{j=6}^{n} p_{j}^{2}}{K_{5}-p_{5}^{2}} \cdot d p_{5}^{2}=g_{p_{5} p_{5}} \cdot d p_{5}^{2}
$$

For more than 7-dimensional space-time surface, according to the curved orthogonal coordinate system we obtain that $g_{f_{6} f_{j}}=0$, for each $j>7$.

By (4),(6),(12),(16) and (19) we obtain the second extra-coordinate 


$$
X_{6}=\sqrt{\mp K_{t} \cdot K_{5} \cdot\left[p_{6}^{2}\left(f_{6}\right)-K_{6}\right]} \cdot \prod_{j=7}^{n} p_{j}\left(f_{j}\right)
$$

(Appendix E) and by defining from now on that $K_{j} \equiv 1$ for each $j \geq 5$, we obtain

$$
g_{f_{6} f_{6}} \cdot d f_{6}^{2}=\sum_{i=1}^{6}\left(\frac{\partial X_{i}}{\partial f_{6}}\right)^{2} d f_{6}^{2}=\frac{K_{t} \cdot \prod_{j=7}^{n} p_{j}^{2}}{1-p_{6}^{2}} \cdot d p_{6}^{2}=g_{p_{6} p_{6}} \cdot d p_{6}^{2}
$$

In this way we can go on and obtain more extra-coordinate equations and extra-metric component equations in which according to (20) and (22), the extra-parameters $f_{j}$ can be changed into $p_{j}$

By (18), (19) and (21) where $K_{j} \equiv 1$ and for each $j \geq 5$, the curved multidimensional space-time surface's equation becomes

$$
\begin{aligned}
\sum_{i=1}^{4} X_{i}^{2} & -X_{t}^{2}=K_{t} \cdot \prod_{j=5}^{n} p_{j}^{2}=\mp X_{5}^{2}+K_{t} \cdot \prod_{j=6}^{n} p_{j}^{2}=\mp X_{5}^{2} \mp X_{6}^{2}+K_{t} \cdot \prod_{j=7}^{n} p_{j}^{2}= \\
& =\sum_{j=5}^{n-2} \mp X_{j}^{2}+K_{t} \cdot p_{n-1}^{2} \cdot p_{n}^{2}=\mp \sum_{j=5}^{n-2} X_{j}^{2} \mp X_{n-1}^{2} \cdot \frac{K_{t} \cdot p_{n}^{2}}{K_{t} \cdot p_{n}^{2}+B_{n}}+K_{t} \cdot p_{n}^{2}
\end{aligned}
$$

where according to (19) and (21), we can write for each $5 \leq j \geq n-2$ the following coordinates equation

$$
X_{j}=\sqrt{ \pm K_{t} \cdot\left(1-p_{j}^{2}\right)} \cdot \prod_{m=j+1}^{n} p_{m}
$$

and

$$
X_{n-1}=\sqrt{\mp\left(p_{n-1}^{2}-1\right) \cdot\left(K_{t} \cdot p_{n}^{2}+B_{n}\right)} \quad, \quad X_{n}=X_{n}\left(p_{n}\right)
$$

in which $B_{n}, B_{6}$ are constant values (Appendix F).

The metric's equation for $n \geq 7$ becomes

$$
\begin{aligned}
& d S_{n}^{2}=-K_{t} \cdot d t^{2} \cdot \prod_{j=5}^{n} p_{j}^{2}+K_{t} \cdot \cosh ^{2}\left(\frac{t}{K_{t}}\right) \cdot d \Omega_{3}^{2} \cdot \prod_{j=5}^{n} p_{j}^{2}+\sum_{j=5}^{n-2} g_{p_{j} p_{j}} \cdot d p_{j}^{2}+ \\
& +g_{p_{n-1} p_{n-1}} \cdot d p_{n-1}^{2}+g_{p_{n} p_{n}} \cdot d p_{n}^{2}
\end{aligned}
$$

where

$$
g_{p_{j} p_{j}}=\frac{K_{t} \cdot \prod_{m=j+1}^{n} p_{m}^{2}}{1-p_{j}^{2}} \quad, \quad g_{p_{n-1} p_{n-1}}=\sum_{i=1}^{n-1}\left(\frac{\partial X_{i}}{\partial p_{n-1}}\right)^{2}=\frac{K_{t} \cdot p_{n}^{2}+B_{n} \cdot p_{n-1}^{2}}{1-p_{n-1}^{2}}
$$

and

$$
g_{p_{n} p_{n}}=\sum_{i=1}^{n}\left(\frac{\partial X_{i}}{\partial p_{n}}\right)^{2}=\frac{K_{t} \cdot B_{n} \cdot p_{n-1}^{2}+K_{t}^{2} \cdot p_{n}^{2}}{K_{t} \cdot p_{n}^{2}+B_{n}} \pm\left(\frac{d X_{n}}{d p_{n}}\right)^{2}
$$

\section{The last extra-coordinate and the final curved multidimensional surface equation by the equivalence principle}

According to the geodesic equations in multidimensional space-time surface, for a local point on a symmetric 
sphere surface and $n-2$ co-moving coordinates of each $n-1$ dimensional space-time surface embedded in a $n$ dimensional space-time surface

Where $x_{i=1,2,3,4}=$ const. and $p_{j=5, \ldots, n-1}=$ const., we obtain that

$$
\frac{d^{2} t}{d \tau^{2}}+\frac{2}{p_{n}} \cdot \frac{d p_{n}}{d \tau} \cdot \frac{d t}{d \tau}=0
$$

or

$$
\left(\frac{d t}{d \tau}\right)^{-1} \cdot d\left(\frac{d t}{d \tau}\right)=-2 \frac{d p_{n}}{p_{n}}
$$

that by integration becomes

$$
\frac{d t}{d \tau}=\frac{C}{p_{n}^{2}}=\frac{1}{\sqrt{K_{t} \cdot \prod_{j=5}^{n} p_{j}^{2}-g_{p_{n} p_{n}} \cdot \frac{d p_{n}^{2}}{d t^{2}}}}
$$

or

$$
\frac{d p_{n}^{2}}{d t^{2}}=\frac{K_{t} \cdot \prod_{j=5}^{n} p_{j}^{2}-\frac{p_{n}^{4}}{C^{2}}}{g_{p_{n} p_{n}}}
$$

We assume that there is at least one geodesic which represent a straight line in the curved multidimensional space-time surface (this is a kind of "equivalence principle" of a new type (E. Guendelman and H. Ruchvarger, 2006), in which

$$
\frac{d^{2} X_{n}}{d \tau^{2}}=0
$$

Thus, by (30), (31), (32) and according to the co-moving coordinates of $n-2$ dimensional space surfaces embedded in the $n$-dimensional space-time surface, we obtain that

$$
\begin{aligned}
& K \equiv\left(\frac{d X_{n}}{d \tau}\right)^{2}=\left(\frac{d X_{n}}{d p_{n}} \cdot \frac{d p_{n}}{d \tau}\right)^{2}=\left(\frac{d X_{n}}{d p_{n}} \cdot \frac{d p_{n}}{d t} \cdot \frac{C}{p_{n}^{2}}\right)^{2} \\
& \left(\frac{d X_{n}}{d p_{n}}\right)^{2}=\frac{B_{n} \cdot p_{n-1}^{2}+K_{t} \cdot p_{n}^{2}}{K_{t} \cdot p_{n}^{2}+B_{n}} \cdot \frac{K \cdot K_{t} \cdot p_{n}^{2}}{K_{t} \cdot C^{2} \cdot \prod_{j=5}^{n-1} p_{j}^{2}-p_{n}^{2} \mp K \cdot p_{n}^{2}}= \\
& =\frac{B_{n} \cdot K^{\prime \prime}+K_{t} \cdot p_{n}^{2}}{K_{t} \cdot p_{n}^{2}+B_{n}} \cdot \frac{K \cdot K_{t} \cdot p_{n}^{2}}{K_{t} \cdot C^{2} \cdot K^{\prime}-(1 \pm K) \cdot p_{n}^{2}}
\end{aligned}
$$

and

$$
d X_{n}=\sqrt{K \cdot K_{t}} \cdot \sqrt{\frac{B_{n} \cdot K^{\prime \prime}+K_{t} \cdot p_{n}^{2}}{\left[K_{t} \cdot p_{n}^{2}+B_{n}\right] \cdot\left[K_{t} \cdot C^{2} \cdot K^{\prime}-(1 \pm K) \cdot p_{n}^{2}\right]}} \cdot p_{n} \cdot d p_{n}
$$

where $K, K^{\prime}$ and $K^{\prime \prime}$ are constant positive values (Appendix $\mathrm{G}$ ).

For $K^{\prime \prime} \equiv 1$, we obtain by the integration of (36), the last extra-coordinate as follows: 


$$
\begin{aligned}
& \int_{0}^{X_{n}} d X_{n}^{\prime}=X_{n}=\sqrt{K \cdot K_{t}} \cdot \int_{C \cdot \sqrt{\frac{K_{t} \cdot K^{\prime}}{1 \pm K}}}^{p_{n}} \frac{p_{n}^{\prime} \cdot d p_{n}^{\prime}}{\sqrt{K_{t} \cdot C^{2} \cdot K^{\prime}-(1 \pm K) \cdot p_{n}^{\prime 2}}}= \\
& =\mp \frac{\sqrt{K \cdot K_{t}}}{1 \pm K} \cdot \sqrt{K_{t} \cdot C^{2} \cdot K^{\prime}-(1 \pm K) \cdot p_{n}^{2}}
\end{aligned}
$$

and

$$
p_{n}^{2}=\frac{K_{t} \cdot K^{\prime} \cdot C^{2}}{1 \pm K}-\frac{1 \pm K}{K \cdot K_{t}} \cdot X_{n}^{2}
$$

Thus, by (23) and (38), the final curved multidimensional surface's equation becomes:

$$
\sum_{i=1}^{4} X_{i}^{2}-X_{t}^{2}=\mp \sum_{j=5}^{n-2} X_{j}^{2} \mp X_{n-1}^{2} \cdot \frac{A_{1}-A_{2} \cdot X_{n}^{2}}{A_{3}-A_{2} \cdot X_{n}^{2}}+\frac{A_{1}-A_{2} \cdot X_{n}^{2}}{A_{4}}
$$

and the curved 6-dimensional surface's equation, becomes

$$
\sum_{i=1}^{4} X_{i}^{2}-X_{t}^{2}=\mp X_{5}^{2} \cdot \frac{A_{1}-A_{2} \cdot X_{6}^{2}}{A_{3}-A_{2} \cdot X_{6}^{2}}+\frac{A_{1}-A_{2} \cdot X_{6}^{2}}{A_{4}}
$$

Where $A_{1} \equiv K \cdot K_{t}^{3} \cdot K^{\prime} \cdot C^{2} \quad, \quad A_{2} \equiv K_{t} \cdot(1 \pm K)^{2} \quad, \quad A_{3} \equiv A_{1}+B_{n} \cdot K \cdot K_{t} \cdot(1 \pm K)$ or $A_{3} \equiv A_{1}+B_{6} \cdot K \cdot K_{t} \cdot(1 \pm K)$ and $A_{4} \equiv K \cdot K_{t} \cdot(1 \pm K)$.

\section{Velocity and acceleration of $3 \mathrm{D}$ particles by the equivalence principles}

According to Einstein's equivalence principle in general relativity, we obtain the differential equation of the geodesic

$$
\frac{d^{2} t}{d \tau^{2}}+\sum_{j=5}^{n} \frac{2}{p_{j}} \cdot \frac{d p_{j}}{d \tau} \cdot \frac{d t}{d \tau}=0
$$

for a local point on a symmetric three-sphere surface where $x_{i=1,2,3,4}=$ const. (9) that by integration becomes

$$
\frac{d t}{d \tau}=\frac{C}{\prod_{j=5}^{n} p_{j}^{2}}
$$

where $c=$ const. and $C \equiv c^{2}=$ const. $>0$.

By (17), (42) and according to the new version for the equivalence principle (33), we obtain for particles with a geodesic constant $\mathrm{C}$, the following equations:

$$
\frac{d \sqrt{\sum_{i=1}^{4} X_{i}^{2}}}{d t}=k \cdot \frac{d \tau}{d t}=\frac{k \cdot \prod_{j=5}^{n} p_{j}^{2}}{C}>0
$$

and

$$
\frac{d^{2} \sqrt{\sum_{i=1}^{4} X_{i}^{2}}}{d t^{2}}=\frac{ \pm 2 k}{C} \cdot \prod_{j=5}^{n} p_{j}^{2} \cdot \sqrt{1+\frac{2 B}{C^{2}} \cdot \prod_{j=5}^{n} p_{j}^{2}}
$$

where $\mathrm{k}, \mathrm{B}$ are constant values and $\mathrm{k}>0$ (Appendix $\mathrm{H})$.

Thus, the $3 \mathrm{D}$ particles velocity represented by (43) is an expanding velocity and according to (44), there are accelerating expanding 3D particles that explains the universe's accelerated expansion. 


\section{Conclusions}

Multidimensional space-times are represented as curved surfaces embedded in higher dimensional flat spaces. The embedding of each surface is based on geometrical principles.

Each new coordinate depends on a new parameter and does not depend on the former parameters. Thus, each coordinate depend on the parameters as separated variables.

Each new parameter is an inverse function of the new coordinate and orthogonally of the former coordinates parameters, means that the curved multidimensional metric will not have off-diagonal components and this gives a condition on embedding functions.

By using variable separated coordinates and orthogonal parameters as curved coordinates, equations are obtained, solving the metric components and we obtain that the universe expands and that the expansion is accelerated. By using co-moving $\mathrm{n}-2$ coordinates in each $\mathrm{n}-1$ dimensional curved space-time surface, in the $\mathrm{n}$-dimensional curved space-time surface assuming that there is at least one geodesic which represent a straight line in the curved multidimensional space-time surface (this is a kind of "equivalence principle" of a new type), we obtain the curved multidimensional space-time surface's equation, its metric and accelerated expanded three-sphere surface's particles that explains the accelerated expansion of the universe, based on geometrical principles rather than specific dynamical equations.

In our opinion, the best explanation for something that is unknown, is by using extra-dimensions. Therefore, we explained the universe accelerated expansion by using extra-dimensions (E. Guendelman and H. Ruchvarger, 2004).

In (E. Guendelman and H. Ruchvarger, 2004), we explain the universe accelerated expansion by defining a general orthogonal multidimensional variable separated coordinate system based on a three-sphere surface.

In (E. Guendelman and H. Ruchvarger, 2004), we find the extra-dimensional metric component by a new equivalence principle that is an additional principle to Einstein's equivalence principle.

But by that new equivalence principle, we find only the first extra-dimensional metric component and to explain the universe acceleration expansion by an orthogonal multidimensional variable separated coordinate system, we need at least two extra-dimensions.

In this article, we find the orthogonal multidimensional variable separated coordinate system that represents the embedded curved multidimensional space-time surface, by a special form of equations for the coordinates, based on geometrical principles.

By this orthogonal multidimensional variable separated coordinate system, we obtain that the universe expands and that the expansion is accelerated, without the need of considering the energy density of the vacuum as in other studies (Y.B. Zeldovich,1967, Paul K. Townsend and Mattias N.R.Wohlfarth,2003, Mauricio Bellini, 2006) and (P.S. Wesson, 1999, Tomas Liko and Paul S. Wesson, 2005).

By this orthogonal curved multidimensional space-time surface coordinates, we also obtain the three-sphere surface coordinates, the metric components of all dimensions, including the extra-dimensions and the curved multidimensional space-time surface equation, for all the extra-dimensions that we want to use.

According to (40), for each last space-like extra-dimension comparing with (E. Guendelman and H. Ruchvarger, 2006) where $n=5$,we obtain a very similar solution as follows:

$$
g_{n n}=\frac{K_{t} \cdot\left(K_{t} \cdot C^{2} \cdot K^{\prime}-p_{n}^{2}\right)}{K_{t} \cdot C^{2} \cdot K^{\prime}-(1+K) \cdot p_{n}^{2}} \quad, \quad g_{f f}=\frac{A \cdot\left(C^{2}-f^{2}\right)}{C^{2}-(B+1) \cdot f^{2}}=\frac{A \cdot\left(C^{2}-f^{2}\right)}{C^{2}-\lambda \cdot f^{2}} \quad, \quad \lambda>1
$$

Thus, for each last space-like extra-dimension comparing with (E. Guendelman and H. Ruchvarger, 2006) where $n=5$, we obtain a very similar solution.

\section{References}

E. Guendelman and H. Ruchvarger. (2004). Int. J. Mod. Phys. A19: 3377 http://dx.doi.org/10.1142/S0217751X04019305

E. Guendelman and H. Ruchvarger. (2006). Foundation of Physics, 36, 1846-1868 http://dx.doi.org/10.1142/S0217751X04019305

E.A. Bergshoff, A. Collinucci, D. Roest, J.G. Russo and P.K. Townsend. (2005). hep-th/0504011 
E.I. Guendelman and A.B. Kaganovich. (2004). gr-qc/0408026v1

E.I. Guendelman and A.B. Kaganovich. (2004). hep-th /041118v2

J. Hartnett. (2005). International Journal of Theoretical Physics.

K.Purohit, Bhatt and Yogesh. (2011). Static Extra Dimension and Ecceleration of the Univers, International Journal of Theoretical Physics, Vol. 50, Number 5.

L.V. Nirop and C.P. Burgess. (2011). Inflation from Codimension-2 Brane Back-reaction, arXiv:1108.2553v1 [hep-th].

M. Carmeli. (2002). Cosmological Spacial Relativity, World Scientific. http://dx.doi.org/10.1142/9789812777553 Mauricio Bellini. (2006). gr-qc/0602014 v1

P.S. Wesson. Space-Time-Matter (1999).World Scientific, Singapore,

Paul K. Townsend and N.R. Wohlfarth. (2003). For application of Kaluza-Klein theories to obtain an accelerated universe, Phys. Rev. Lett. 91:06130

Paul K.Townsend and Mattias N.R.Wohlfarth. (2003). Puys. Rev. Lett. 91:061302

S. Behar and M. Carmeli. (2000). Journal-ref: Int.J.Theor.Phys. 39

T.Appelquist, A.Chodos and P.G.O. (1987). Freund,Modern Kaluza-KleinTheories.

Tomas Liko and Paul S. Wesson. (2005). gr-qc/0505024 v2

Y. Kim, C. Young Oh and N. Park. (2002). hep-th/0212326

Y.B. Zeldovich. (1967). JETP Lett. 6:316, Pisma Zh. Eksp. Teor. 6:883

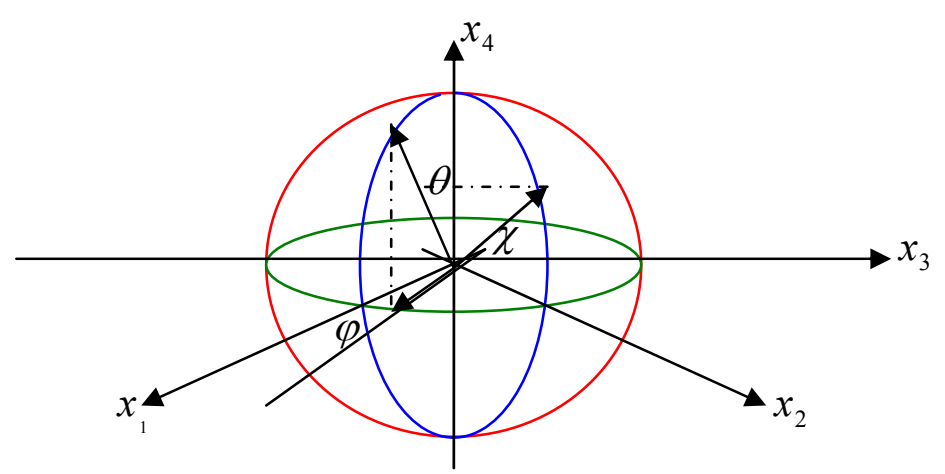

Figure 1. A hyper-sphere or a three sphere surface represented by the coordinates:

$$
\begin{aligned}
& x_{1}=\sin (\chi) \cdot \sin (\theta) \cdot \cos (\varphi) \quad, \quad x_{2}=\sin (\chi) \cdot \sin (\theta) \cdot \sin (\varphi), \\
& x_{3}=\sin (\chi) \cdot \cos (\theta) \quad \text { and } \quad x_{4}=\cos (\chi)
\end{aligned}
$$

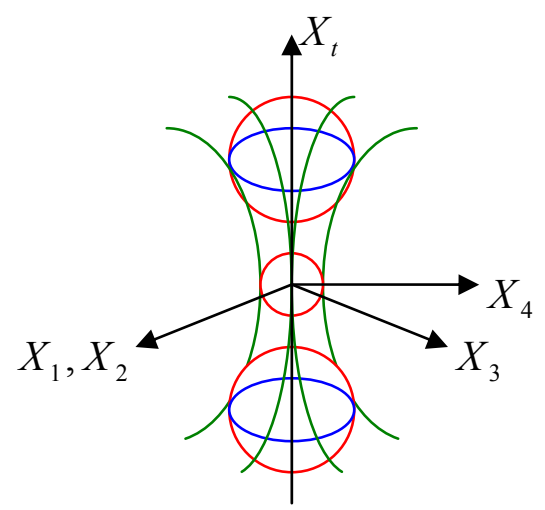

Figure 2. Hyperbolic four-Dimensional space-time universe embedding infinite three-sphere surfaces represented by equation (18) 
Appendix A-Proof that surface's metric, represented by a curved orthogonal coordinates system, has no off-diagonal component

For a curved multidimensional surface represented by curved coordinates $(u, v$,$) and embedded in a$ higher-dimensional hyper-flat space with a orthogonal coordinate system $x_{i}$, each point is represented by the vector

$$
\vec{r}=\sum_{i=1}^{n} x_{i}(u, v, \ldots) \hat{x}_{i}
$$

in which the vector

$$
d \vec{r}=\sum_{i=1}^{n} d x_{i}(u, v, \ldots) \hat{x}_{i}
$$

is tangential to the embedded surface.

Vectors, tangential to the embedded surface in the parameter direction, means $d \vec{r}(u)$ in the parameter $u$ direction where except of $u$ all other parameters are constant and $d \vec{r}(v)$ in the parameter $v$ direction where except of $v$ all other parameters are constant, are represented by

$$
d \vec{r}(u)=\sum_{i=1}^{n} d x_{i}(u) \cdot \hat{x}_{i}=\sum_{i=1}^{n} \frac{\partial x_{i}}{\partial u} d u \cdot \hat{x}_{i}
$$

and

$$
d \vec{r}(v)=\sum_{i=1}^{n} d x_{i}(v) \cdot \hat{x}_{i}=\sum_{i=1}^{n} \frac{\partial x_{i}}{\partial v} d v \cdot \hat{x}_{i}
$$

The angle between the vectors is solved by the equation

$$
\begin{gathered}
\cos (u, v)=\frac{d \vec{r}(u) \bullet d \vec{r}(v)}{|d \vec{r}(u)| \cdot d \vec{r}(v) \mid}=\frac{\sum_{i=1}^{n} \frac{\partial x_{i}}{\partial u} d u \cdot \hat{x}_{i} \cdot \sum_{i=1}^{n} \frac{\partial x_{i}}{\partial v} d v \cdot \hat{x}_{i}}{\sqrt{\left(\sum_{i=1}^{n} \frac{\partial x_{i}}{\partial u} d u\right)^{2}} \cdot \sqrt{\left(\sum_{i=1}^{n} \frac{\partial x_{i}}{\partial v} d v\right)^{2}}}= \\
=\frac{\sum_{i=1}^{n} \frac{\partial x_{i}}{\partial u} \cdot \frac{\partial x_{i}}{\partial v}}{\sqrt{\left(\sum_{i=1}^{n} \frac{\partial x_{i}}{\partial u}\right)^{2} \cdot\left(\sum_{i=1}^{n} \frac{\partial x_{i}}{\partial v}\right)^{2}}}=\frac{\eta_{\alpha \beta} \frac{\partial x^{\alpha}}{\partial u} \cdot \frac{\partial x^{\beta}}{\partial v}}{\sqrt{\eta_{\alpha \beta} \frac{\partial x^{\alpha}}{\partial u} \cdot \frac{\partial x^{\beta}}{\partial u} \cdot \eta_{\alpha \beta} \frac{\partial x^{\alpha}}{\partial v} \cdot \frac{\partial x^{\beta}}{\partial v}}}=\frac{g_{u v}}{\sqrt{g_{u u} \cdot g_{v v}}}
\end{gathered}
$$

Thus,

$$
d \vec{r}(u) \perp d \vec{r}(v) \Leftrightarrow \cos (u, v)=\cos \left(\frac{\pi}{2}\right) \Leftrightarrow g_{u v}=0
$$

which means that the metric of a surface represented by curved orthogonal coordinates, will not have off-diagonal components.

Appendix B- 3D space coordinates components equations, calculated by variable separate coordinates and metric without off-diagonal components

By (3) and according to the orthogonal coordinate system, we obtain for each $m \geq 5$, the following equations 


$$
\begin{aligned}
& g_{\varphi f_{m}}=g_{\varphi t}=g_{\varphi \chi}=0=g_{\varphi \theta}=2 \sum_{i=1}^{2} \frac{\partial X_{i}}{\partial \varphi} \cdot \frac{\partial X_{i}}{\partial \theta}= \\
& =2 p_{1 t}^{2} \cdot p_{1 \chi}^{2} \cdot p_{1 \theta} \cdot p_{1 \varphi} \cdot \frac{d p_{1 \varphi}}{d \varphi} \cdot \frac{d p_{1 \theta}}{d \theta} \cdot \prod_{m=5}^{n} p_{1 m}^{2}+ \\
& +2 p_{2 t}^{2} \cdot p_{2 \chi}^{2} \cdot p_{2 \theta} \cdot p_{2 \varphi} \cdot \frac{d p_{2 \varphi}}{d \varphi} \cdot \frac{d p_{2 \theta}}{d \theta} \cdot \prod_{m=5}^{n} p_{2 m}^{2}
\end{aligned}
$$

in which

$$
\frac{p_{1 t}^{2} \cdot p_{1 \chi}^{2} \cdot p_{1 \theta} \cdot d p_{1 \theta} \cdot \prod_{m=5}^{n} p_{1 m}^{2}}{p_{2 t}^{2} \cdot p_{2 \chi}^{2} \cdot p_{2 \theta} \cdot d p_{2 \theta} \cdot \prod_{m=5}^{n} p_{2 m}^{2}}=-\frac{p_{2 \varphi} \cdot d p_{2 \varphi}}{p_{1 \varphi} \cdot d p_{1 \varphi}}=\text { const } .=1
$$

that by integration becomes

$$
-p_{2 \varphi}^{2}=p_{1 \varphi}^{2}-1
$$

and also

$$
\frac{p_{1 t}^{2} \cdot p_{1 \chi}^{2} \cdot \prod_{m=5}^{n} p_{1 m}^{2}}{p_{2 t}^{2} \cdot p_{2 \chi}^{2} \cdot \prod_{m=5}^{n} p_{2 m}^{2}}=\frac{p_{2 \theta} \cdot d p_{2 \theta}}{p_{1 \theta} \cdot d p_{1 \theta}}=\text { const. }=1
$$

in which

$$
p_{2 \theta}=p_{1 \theta}, p_{2 \chi}=p_{1 \chi}, p_{2 t}=p_{1 t}
$$

and

$$
\prod_{m=5}^{n} p_{2 m}^{2}=\prod_{m=5}^{n} p_{1 m}^{2}
$$

In the same way, by (3) and according to the orthogonal coordinate system, we obtain for each $m \geq 5$, the following equations

$$
\begin{aligned}
& g_{\theta f_{m}}=g_{\theta t}=0=g_{\theta \chi}=2 \sum_{i=1}^{3} \frac{\partial X_{i}}{\partial \chi} \cdot \frac{\partial X_{i}}{\partial \theta}= \\
& =2 p_{1 t}^{2} \cdot p_{1 \theta} \cdot p_{1 \chi} \cdot \frac{d p_{1 \chi}}{d \chi} \cdot \frac{d p_{1 \theta}}{d \theta} \cdot\left(p_{1 \varphi}^{2}+p_{2 \varphi}^{2}\right) \cdot \prod_{m=5}^{n} p_{1 m}^{2}+ \\
& +2 p_{3 t}^{2} \cdot p_{3 \chi} \cdot p_{3 \theta} \cdot \frac{d p_{3 \chi}}{d \chi} \cdot \frac{d p_{3 \theta}}{d \theta} \cdot \prod_{m=5}^{n} p_{3 m}^{2}
\end{aligned}
$$

in which

$$
\frac{p_{1 t}^{2} \cdot p_{1 \chi} \cdot d p_{1 \chi} \cdot \prod_{m=5}^{n} p_{1 m}}{p_{3 t}^{2} \cdot p_{3 \chi} \cdot d p_{3 \chi} \cdot \prod_{m=5}^{n} p_{3 m}}=-\frac{p_{3 \theta} \cdot d p_{3 \theta}}{p_{1 \theta} \cdot d p_{\theta}}=\text { const }=1
$$

that by integration becomes

$$
-p_{3 \theta}^{2}=p_{1 \theta}^{2}-1
$$


and also

$$
\frac{p_{1 t}^{2} \cdot \prod_{m=5}^{n} p_{1 m}^{2}}{p_{2 t}^{2} \cdot \prod_{m=5}^{n} p_{2 m}^{2}}=\frac{p_{3 \chi} \cdot d p_{3 \chi}}{p_{1 \chi} \cdot d p_{1 \chi}}=\text { const. }=1
$$

in which

$$
p_{3 \chi}=p_{1 \chi}, p_{3 t}=p_{1 t}
$$

and

$$
\prod_{m=5}^{n} p_{3 m}^{2}=\prod_{m=5}^{n} p_{1 m}^{2}
$$

In the same way, by (3) and according to the orthogonal coordinate system, we obtain for each $m \geq 5$, the following equations

$$
\begin{aligned}
& g_{f_{m} \chi}=0=g_{t \chi}=2 \sum_{i=1}^{4} \frac{\partial X_{i}}{\partial \chi} \cdot \frac{\partial X_{i}}{\partial t}=2 p_{1 t} \cdot p_{1 \chi} \cdot \frac{d p_{1 \chi}}{d \chi} \cdot \frac{d p_{1 t}}{d t} \cdot\left(p_{1 \theta}^{2}+p_{3 \theta}^{2}\right) \cdot \prod_{m=5}^{n} p_{1 m}^{2}+ \\
& +2 p_{4 \chi} \cdot p_{4 t} \cdot \frac{d p_{4 \chi}}{d \chi} \cdot \frac{d p_{4 t}}{d t} \cdot \prod_{m=5}^{n} p_{4 m}^{2}
\end{aligned}
$$

in which

$$
\frac{p_{1 t} \cdot d p_{1 t} \cdot \prod_{m=5}^{n} p_{1 m}}{p_{4 t} \cdot d p_{4 t} \cdot \prod_{m=5}^{n} p_{4 m}}=-\frac{p_{4 \chi} \cdot d p_{4 \chi}}{p_{1 \chi} \cdot d p_{4 \chi}}=\text { const }=1
$$

that by integration becomes

$$
-p_{4 \chi}^{2}=p_{1 \chi}^{2}-1
$$

and also

$$
\frac{\prod_{m=5}^{n} p_{1 m}^{2}}{\prod_{m=5}^{n} p_{2 m}^{2}}=\frac{p_{4 t} \cdot d p_{4 t}}{p_{1 t} \cdot d p_{1 t}}=\text { const. }=1
$$

in which

$$
p_{4 t}=p_{1 t}
$$

and

$$
\prod_{m=5}^{n} p_{4 m}^{2}=\prod_{m=5}^{n} p_{1 m}^{2}
$$

Appendix C- 4D space-time coordinates equations, calculated by variable separate coordinates and metric without off-diagonal components

According to the orthogonal coordinate system and by (3),(6), in the same way as we obtain the former coordinates, we obtain for each $m \geq 5$ the following equation 


$$
\begin{aligned}
& g_{t f_{m}}=0=2 \sum_{i=1}^{4} \frac{\partial X_{i}}{\partial f_{m}} \cdot \frac{\partial X_{i}}{\partial t}-2 \frac{\partial X_{t}}{\partial f_{m}} \cdot \frac{\partial X_{t}}{\partial t}=2 A \cdot p_{1 \chi}^{2} \cdot p_{1 t} \cdot \frac{d p_{1 t}}{d t} \cdot \frac{\partial}{\partial f_{m}} \prod_{m=5}^{n} p_{1 m}^{2}+ \\
& +2 p_{4 \chi}^{2} \cdot p_{4 t} \cdot \frac{d p_{4 t}}{d t} \cdot \frac{\partial}{\partial f_{m}} \prod_{m=6}^{n} p_{4 m}^{2}-2 A \cdot p_{t t} \cdot \frac{d p_{t t}}{d t} \cdot \frac{\partial}{\partial f_{m}} \prod_{m=5}^{n} p_{t m}^{2}= \\
& =2 p_{t} \cdot \frac{d p_{t}}{d t} \cdot \frac{\partial}{\partial f_{m}} \prod_{m=5}^{n} p_{m}^{2}-2 A \cdot p_{t t} \cdot \frac{d p_{t t}}{d t} \cdot \frac{\partial}{\partial f_{m}} \prod_{m=5}^{n} p_{t m}^{2}
\end{aligned}
$$

that becomes

$$
\frac{\frac{\partial}{\partial f_{m}} \prod_{m=5}^{n} p_{m}^{2}}{\frac{\partial}{\partial f_{m}} \prod_{m=5}^{n} p_{t m}^{2}}=\frac{p_{m} \cdot d p_{m} \cdot p_{t m}^{2} \cdot \prod_{m=5}^{n} p_{m}^{2}}{p_{t m} \cdot d p_{t m} \cdot p_{m}^{2} \cdot \prod_{m=5}^{n} p_{t m}^{2}}=\frac{p_{t t} \cdot d p_{t t}}{p_{t} \cdot d p_{1 t}}=\text { const. } \equiv C_{t}
$$

and for each $k, m \geq 5$, becomes

$$
\frac{p_{m} \cdot d p_{m} \cdot p_{t m}^{2} \cdot p_{t k}^{2} \cdot \prod_{m=5}^{n} p_{m}^{2}}{p_{t m} \cdot d p_{t m} \cdot p_{m}^{2} \cdot p_{k}^{2} \cdot \prod_{m=5}^{n} p_{t m}^{2}}=\frac{C_{t} \cdot p_{t k}^{2}}{p_{k}^{2}}=\text { const. } \equiv C_{t k}
$$

By integration of (72) and by (73), we obtain the following equations

$$
p_{t t}^{2}=C_{t} \cdot p_{t}^{2}-B_{t} \quad, \quad C_{t} \cdot \prod_{m=5}^{n} p_{t m}^{2}=\prod_{m=5}^{n} p_{m}^{2}
$$

where $B_{t}=$ const.

Appendix D-First extra-coordinate calculated by variable separate coordinates and metric without off-diagonal components

According to the orthogonal coordinate system and by (3), (6), (12), (16), in the same way as we obtain the former coordinates, we obtain for each $j>6$ the following equation

$$
\begin{aligned}
& g_{f_{5} f_{j}}=0=2\left(-\frac{\partial X_{t}}{\partial f_{5}} \cdot \frac{\partial X_{t}}{\partial f_{j}}+\sum_{m=1}^{4} \frac{\partial X_{m}}{\partial f_{5}} \cdot \frac{\partial X_{m}}{\partial f_{j}} \pm \frac{\partial X_{5}}{\partial f_{5}} \cdot \frac{\partial X_{5}}{\partial f_{j}}\right)= \\
& =\frac{2 K_{t} \cdot p_{5} \cdot p_{j} \cdot \prod_{m=6}^{n} p_{m}^{2}}{p_{j}^{2}} \cdot \frac{d p_{5}}{d f_{5}} \cdot \frac{d p_{j}}{d f_{j}} \pm \frac{2 p_{55} \cdot p_{5 j} \cdot \prod_{m=6}^{n} p_{5 m}^{2}}{p_{5 j}^{2}} \cdot \frac{d p_{55}}{d f_{5}} \cdot \frac{d p_{5 j}}{d f_{j}}
\end{aligned}
$$

that becomes

$$
\frac{K_{t} \cdot p_{j} \cdot d p_{j} \cdot p_{5 j}^{2} \cdot \prod_{m=6}^{n} p_{m}^{2}}{p_{5 j} \cdot d p_{5 j} \cdot p_{j}^{2} \cdot \prod_{m=6}^{n} p_{5 m}^{2}}=\mp \frac{p_{55} \cdot d p_{55}}{p_{5} \cdot d p_{5}}=C_{5}=\text { const } \text {. }
$$

and for each $i, j \geq 6$, becomes 


$$
\frac{K_{t} \cdot p_{j} \cdot d p_{j} \cdot p_{5 j}^{2} \cdot p_{5 i}^{2} \cdot \prod_{m=6}^{n} p_{m}^{2}}{p_{5 j} \cdot d p_{5 j} \cdot p_{j}^{2} \cdot p_{i}^{2} \cdot \prod_{m=6}^{n} p_{5 m}^{2}}=C_{5} \cdot \frac{p_{5 i}^{2}}{p_{i}^{2}}=C_{i p}=\text { const } \text {. }
$$

By integration of (74) and by (75), we obtain

$$
\mp P_{55}^{2}\left(f_{5}\right)=C_{5} \cdot p_{5}^{2}\left(f_{5}\right)-B_{5}
$$

and

$$
K_{t} \cdot \prod_{m=6}^{n} p_{m}^{2}\left(f_{m}\right)=C_{5} \cdot \prod_{m=6}^{n} p_{5 m}^{2}\left(f_{m}\right)
$$

By substituting (76) and (77) in (4), we obtain the first extra-coordinate

$$
X_{5}=p_{55}\left(f_{5}\right) \cdot \prod_{j=6}^{n} p_{5 j}\left(f_{j}\right)=\sqrt{\mp K_{t} \cdot\left[p_{5}^{2}\left(f_{5}\right)-K_{5}\right]} \cdot \prod_{j=6}^{n} p_{j}\left(f_{j}\right)
$$

where $B_{5}=$ const . and $K_{5} \equiv \frac{B_{5}}{C_{5}}$

Appendix E-Second extra-coordinate calculated by variable separate coordinates and metric without off-diagonal components

According to the orthogonal coordinate system and by (3),(6),(12),(16),(19), in the same way as we obtain the former coordinates, we obtain for each $j>7$, the following equation

$$
\begin{aligned}
& g_{f_{6} f_{j}}=0=2\left(-\frac{\partial X_{t}}{\partial f_{6}} \cdot \frac{\partial X_{t}}{\partial f_{j}}+\sum_{m=1}^{4} \frac{\partial X_{m}}{\partial f_{6}} \cdot \frac{\partial X_{m}}{\partial f_{j}} \pm \frac{\partial X_{5}}{\partial f_{6}} \cdot \frac{\partial X_{5}}{\partial f_{j}} \pm \frac{\partial X_{6}}{\partial f_{6}} \cdot \frac{\partial X_{6}}{\partial f_{j}}\right)= \\
& =\frac{2 K_{t} \cdot \prod_{m=5}^{n} p_{m}^{2}}{p_{6} \cdot p_{j}} \cdot \frac{d p_{6}}{d f_{6}} \cdot \frac{d p_{j}}{d f_{j}}-\frac{2\left(K_{t} \cdot p_{5}^{2}-K_{t} \cdot K_{5}\right) \cdot \prod_{m=6}^{n} p_{m}^{2}}{p_{6} \cdot p_{j}} \cdot \frac{d p_{6}}{d f_{6}} \cdot \frac{d p_{j}}{d f_{j}} \\
& \pm \frac{2 \prod_{m=6}^{n} p_{6 m}^{2}}{p_{66} \cdot p_{6 j}} \cdot \frac{d p_{66}}{d f_{6}} \cdot \frac{d p_{6 j}}{d f_{j}}=\frac{2 K_{t} \cdot K_{5} \cdot \prod_{m=6}^{n} p_{m}^{2}}{p_{6} \cdot p_{j}} \cdot \frac{d p_{6}}{d f_{6}} \cdot \frac{d p_{j}}{d f_{j}} \pm \frac{2 \prod_{m=6}^{n} p_{6 m}^{2}}{p_{66} \cdot p_{6 j}} \cdot \frac{d p_{66}}{d f_{6}} \cdot \frac{d p_{6 j}}{d f_{j}}= \\
& =\frac{2 K_{t} \cdot K_{5} \cdot p_{6} \cdot \prod_{m=7}^{n} p_{m}^{2}}{p_{j}} \cdot \frac{d p_{6}}{d f_{6}} \cdot \frac{d p_{j}}{d f_{j}} \pm \frac{2 p_{66} \cdot \prod_{m=7}^{n} p_{6 m}^{2}}{p_{6 j}} \cdot \frac{d p_{66}}{d f_{6}} \cdot \frac{d p_{6 j}}{d f_{j}}
\end{aligned}
$$

that becomes

$$
\frac{K_{t} \cdot K_{5} \cdot p_{6 j}^{2} \cdot p_{j} \cdot d p_{j} \cdot \prod_{m=7}^{n} p_{m}^{2}}{p_{j}^{2} \cdot p_{6 j} \cdot d p_{6 j} \cdot \prod_{m=7}^{n} p_{6 m}^{2}}=\mp \frac{p_{66} \cdot d p_{66}}{p_{6} \cdot d p_{6}}=C_{6}=\text { const } \text {. }
$$

and for each $i, j \geq 7$, becomes 


$$
\frac{K_{t} \cdot K_{5} \cdot p_{6 j} \cdot d p_{j} \cdot p_{6 i}^{2} \cdot \prod_{m=7}^{n} p_{m}^{2}}{p_{j} \cdot p_{6 j} \cdot p_{i}^{2} \cdot \prod_{m=7}^{n} p_{6 m}^{2}}=C_{6} \cdot \frac{p_{6 i}^{2}}{p_{i}^{2}}=C_{i p}=\text { const } \text {. }
$$

By integration of (81) and by (82), we obtain that

$$
\mp P_{66}^{2}\left(f_{6}\right)=C_{6} \cdot p_{6}^{2}\left(f_{6}\right)-B_{6}
$$

and

$$
K_{t} \cdot K_{5} \cdot \prod_{m=7}^{n} p_{m}^{2}\left(f_{m}\right)=C_{6} \cdot \prod_{m=7}^{n} p_{6 m}^{2}\left(f_{m}\right)
$$

By substituting (83) and (84) in (4), we obtain the second extra-coordinate

$$
X_{6}=p_{66}\left(f_{6}\right) \cdot \prod_{j=7}^{n} p_{6 j}\left(f_{j}\right)=\sqrt{\mp K_{t} \cdot K_{5} \cdot\left[p_{6}^{2}\left(f_{6}\right)-K_{6}\right]} \cdot \prod_{j=7}^{n} p_{j}\left(f_{j}\right)
$$

here $B_{6}=$ const. and $K_{6} \equiv \frac{B_{6}}{C_{6}}$.

\section{Appendix F-The calculation of two last extra-coordinates and the space-time expected surface equations}

Refers to (23), in order to obtain $p_{n-1}^{2} \cdot p_{n}^{2}$ as a function of the coordinates $X_{n-1}$ and $X_{n}$, we can decide that $n=7$ and according to the orthogonal coordinate system in which

$$
\begin{aligned}
& g_{p_{6} p_{7}}=0=2\left(-\frac{\partial X_{t}}{\partial p_{6}} \cdot \frac{\partial X_{t}}{\partial p_{7}}+\sum_{m=1}^{4} \frac{\partial X_{m}}{\partial p_{6}} \cdot \frac{\partial X_{m}}{\partial p_{7}} \pm \sum_{j=5}^{6} \frac{\partial X_{j}}{\partial p_{6}} \cdot \frac{\partial X_{j}}{\partial p_{7}}\right)= \\
& =2 K_{t} \cdot p_{5}^{2} \cdot p_{6} \cdot p_{7}-2 K_{t} \cdot\left(p_{5}^{2}-1\right) \cdot p_{6} \cdot p_{7} \pm 2 p_{67} \cdot \frac{d p_{66}}{d p_{6}} \cdot p_{66} \cdot \frac{d p_{67}}{d p_{7}}= \\
& =2 K_{t} \cdot p_{6} \cdot p_{7} \pm 2 p_{67} \cdot \frac{d p_{66}}{d p_{6}} \cdot p_{66} \cdot \frac{d p_{67}}{d p_{7}}
\end{aligned}
$$

we obtain by the separated variable coordinates in (86), the following equations

$$
\frac{K_{t} \cdot p_{7} \cdot d p_{7}}{p_{67} \cdot d p_{67}}=\mp \frac{p_{66} \cdot d p_{66}}{p_{6} \cdot d p_{6}}=C_{6}=\text { const } \text {. }
$$

By the integrations of (87), we obtain the following equations

$$
\mp P_{66}^{2}=C_{6} \cdot p_{6}^{2}-B_{6}
$$

and

$$
\mathrm{K}_{\mathrm{t}} \cdot P_{7}^{2}=C_{6} \cdot p_{67}^{2}-B_{7}
$$

By substituting (88) and (89) in (4), we obtain the coordinate

$$
X_{6}=p_{66} \cdot p_{67}=\sqrt{\mp\left(p_{6}^{2}-1\right) \cdot\left(K_{t} \cdot p_{7}^{2}+B_{7}\right)}
$$

and the curved 7D space-time surface's equation 


$$
\begin{aligned}
& \sum_{i=1}^{4} X_{i}^{2}-X_{t}^{2}=\sum_{j=5}^{5} \mp X_{j}^{2}+K_{t} \cdot \prod_{m=6}^{7} p_{m}^{2}=\mp X_{5}^{2}+K_{t} \cdot p_{6}^{2} \cdot p_{7}^{2}= \\
& =\mp X_{5}^{2} \mp X_{6}^{2}-B_{7} \cdot p_{6}^{2}+K_{t} \cdot p_{7}^{2}+B_{7}=\mp X_{5}^{2} \mp X_{6}^{2}-B_{7} \cdot\left(p_{6}^{2}-1\right)+K_{t} \cdot p_{7}^{2}= \\
& =\mp X_{5}^{2} \mp X_{6}^{2} \pm \frac{B_{7} \cdot X_{6}^{2}}{K_{t} \cdot p_{7}^{2}+B_{7}}+K_{t} \cdot p_{7}^{2}=\mp X_{5}^{2} \mp X_{6}^{2} \cdot \frac{K_{t} \cdot p_{7}^{2}}{K_{t} \cdot p_{7}^{2}+B_{7}}+K_{t} \cdot p_{7}^{2}
\end{aligned}
$$

where $B_{7}=$ const. and

$$
X_{7}=\prod_{j=7}^{7} p_{7 j}\left(p_{j}\right)=p_{77}\left(p_{7}\right)
$$

Appendix G- Last extra-coordinate as function of last curved coordinate, calculated by assuming co-moving coordinates and by the equivalence principles

By substituting (28) in (32), we obtain

$$
\frac{d p_{n}^{2}}{d t^{2}}=\frac{K_{t} \cdot \prod_{j=5}^{n} p_{j}^{2}-\frac{p_{n}^{4}}{C^{2}}}{g_{p_{n} p_{n}}}=\frac{K_{t} \cdot \prod_{j=5}^{n} p_{j}^{2}-\frac{p_{n}^{4}}{C^{2}}}{\frac{K_{t} \cdot B_{n} \cdot p_{n-1}^{2}+K_{t}^{2} \cdot p_{n}^{2}}{K_{t} \cdot p_{n}^{2}+B_{n}} \pm\left(\frac{d X_{n}}{d p_{n}}\right)^{2}}
$$

According to the new version for the equivalence principle (33) and by (34),(93),

we obtain

$$
\begin{aligned}
& K \equiv\left(\frac{d X_{n}}{d \tau}\right)^{2}=\left(\frac{d X_{n}}{d p_{n}} \cdot \frac{d p_{n}}{d \tau}\right)^{2}=\left(\frac{d X_{n}}{d p_{n}} \cdot \frac{d p_{n}}{d t} \cdot \frac{C}{p_{n}^{2}}\right)^{2}= \\
& =\left(\frac{d X_{n}}{d p_{n}}\right)^{2} \cdot \frac{K_{t} \cdot \prod_{j=5}^{n} p_{j}^{2}-\frac{p_{n}^{4}}{C^{2}}}{\frac{K_{t} \cdot B_{n} \cdot p_{n-1}^{2}+K_{t}^{2} \cdot p_{n}^{2}}{K_{t} \cdot p_{n}^{2}+B_{n}} \pm\left(\frac{d X_{n}}{d p_{n}}\right)^{2}} \cdot \frac{C^{2}}{p_{n}^{4}}= \\
& =\left(\frac{d X_{n}}{d p_{n}}\right)^{2} \cdot \frac{K_{t} \cdot C^{2} \cdot \prod_{j=5}^{n-1} p_{j}^{2}-p_{n}^{2}}{\frac{K_{t} \cdot B_{n} \cdot p_{n-1}^{2}+K_{t}^{2} \cdot p_{n}^{2}}{K_{t} \cdot p_{n}^{2}+B_{n}} \cdot p_{n}^{2} \pm p_{n}^{2} \cdot\left(\frac{d X_{n}}{d p_{n}}\right)^{2}} \\
& \left(\frac{d X_{n}}{d p_{n}}\right)^{2}=\frac{B_{n} \cdot p_{n-1}^{2}+K_{t} \cdot p_{n}^{2}}{K_{t} \cdot p_{n}^{2}+B_{n}} \cdot \frac{K \cdot K_{t} \cdot p_{n}^{2}}{K_{t} \cdot C^{2} \cdot \prod_{j=5}^{n-1} p_{j}^{2}-p_{n}^{2} \mp K \cdot p_{n}^{2}}= \\
& =\frac{B_{n} \cdot K^{\prime \prime}+K_{t} \cdot p_{n}^{2}}{K_{t} \cdot p_{n}^{2}+B_{n}} \cdot \frac{K \cdot K_{t} \cdot p_{n}^{2}}{K_{t} \cdot C^{2} \cdot K^{\prime}-(1 \pm K) \cdot p_{n}^{2}}
\end{aligned}
$$

and 


$$
d X_{n}=\sqrt{K \cdot K_{t}} \cdot \sqrt{\frac{B_{n} \cdot K^{\prime \prime}+K_{t} \cdot p_{n}^{2}}{\left[K_{t} \cdot p_{n}^{2}+B_{n}\right] \cdot\left[K_{t} \cdot C^{2} \cdot K^{\prime}-(1 \pm K) \cdot p_{n}^{2}\right]}} \cdot p_{n} \cdot d p_{n}
$$

where $K, K^{\prime}$ and $K^{\prime \prime}$ are constants .

By (28) and (96), for $K^{\prime \prime} \equiv 1$ we also obtain that

$$
g_{p_{n} p_{n}}=\sum_{i=1}^{n}\left(\frac{\partial x_{i}}{\partial p_{n}}\right)^{2}=K_{t} \pm \frac{K \cdot K_{t} \cdot p_{n}^{2}}{K_{t} \cdot C^{2} \cdot K^{\prime}-(1 \pm K) \cdot p_{n}^{2}}=K_{t} \frac{K_{t} \cdot C^{2} \cdot K^{\prime}-p_{n}^{2}}{K_{t} \cdot C^{2} \cdot K^{\prime}-(1 \pm K) \cdot p_{n}^{2}}
$$

Appendix H- Velocity and acceleration of 3D particles in extra-dimensions, calculated by the equivalence principles

According to the new version for the equivalence principle (33) and by (8), (9),(16), (31), we obtain for particles with a geodesic constant $\mathrm{C}$ the following equations

$$
\begin{aligned}
& 0=\frac{d^{2} X_{i}}{d \tau^{2}}=\frac{d^{2}\left[\sqrt{K_{t}} \cdot x_{i} \cdot \prod_{j=5}^{n} p_{j} \cdot \cosh (t)\right]}{d \tau^{2}}=\frac{x_{i} \cdot d^{2} \sqrt{\sum_{i=1}^{4} X_{i}^{2}}}{d \tau^{2}}= \\
& =x_{i} \cdot \frac{d}{d t}\left(\frac{d \sqrt{\sum_{i=1}^{4} X_{i}^{2}}}{d t} \cdot \frac{d t}{d \tau}\right) \cdot \frac{d t}{d \tau}= \\
& =x_{i} \cdot \frac{d^{2} \sqrt{\sum_{i=1}^{4} X_{i}^{2}}}{d t^{2}} \cdot\left(\frac{d t}{d \tau}\right)^{2}+x_{i} \cdot \frac{d \sqrt{\sum_{i=1}^{4} X_{i}^{2}}}{d t} \cdot \frac{d\left(\frac{d t}{d \tau}\right)}{d t} \cdot \frac{d t}{d \tau}= \\
& =\sqrt{K_{t}} \cdot x_{i} \cdot \frac{\left[\frac{d\left(\prod_{j=5}^{n} p_{j}\right)}{d \tau} \cdot \cosh (t)+\frac{C}{\prod_{j=5}^{n} p_{j}} \cdot \sinh (t)\right]}{d \tau}= \\
& =\left[\frac{d^{2}\left(\prod_{j=5}^{n} p_{j}\right)}{d \tau^{2}}+\frac{C^{2}}{\prod_{j=5}^{n} p_{j}^{3}}\right] \cdot \sqrt{K_{t}} \cdot x_{i} \cdot \cosh (t)
\end{aligned}
$$

where $x_{i}, i=1,2,3,4$ are constants.

Thus,

$$
\frac{d^{2} \sqrt{\sum_{i=1}^{4} X_{i}^{2}}}{d t^{2}} \cdot \frac{d t}{d \tau}+\frac{d \sqrt{\sum_{i=1}^{4} X_{i}^{2}}}{d t} \cdot \frac{d\left(\frac{d t}{d \tau}\right)}{d t}=0
$$

and 


$$
d\left(\frac{d \sqrt{\sum_{i=1}^{4} X_{i}^{2}}}{d t}\right) \cdot \frac{d t}{d \tau}+\frac{d \sqrt{\sum_{i=1}^{4} X_{i}^{2}}}{d t} \cdot d\left(\frac{d t}{d \tau}\right)=0
$$

that by integration becomes

$$
\begin{gathered}
d\left(\frac{d \sqrt{\sum_{i=1}^{4} X_{i}^{2}}}{d t}\right) \\
\int \frac{d \sqrt{\sum_{i=1}^{4} X_{i}^{2}}}{d t}=-\int \frac{d\left(\frac{d t}{d \tau}\right)}{\frac{d t}{d \tau}}= \\
=-\ln \frac{d t}{d \tau}+\ln (k)=\ln \left(k \cdot \frac{d \tau}{d t}\right)
\end{gathered}
$$

Thus, the 3D particles velocity is an expanding velocity, according to

$$
\frac{d \sqrt{\sum_{i=1}^{4} X_{i}^{2}}}{d t}=k \cdot \frac{d \tau}{d t}=\frac{k \cdot \prod_{j=5}^{n} p_{j}^{2}}{C}>0
$$

and the $3 \mathrm{D}$ particles acceleration becomes

$$
\frac{d^{2} \sqrt{\sum_{i=1}^{4} X_{i}^{2}}}{d t^{2}}=\frac{2 k}{C} \cdot \prod_{j=5}^{n} p_{j} \cdot \frac{d\left(\prod_{j=5}^{n} p_{j}\right)}{d t}
$$

where $\mathrm{k}=$ constant and $\mathrm{k}>0$.

By (98) we also obtain that,

$$
\frac{d^{2}\left(\prod_{j=5}^{n} p_{j}\right)}{d \tau^{2}}+\frac{C^{2}}{\prod_{j=5}^{n} p_{j}^{3}}=\frac{\left[\frac{d\left(\prod_{j=5}^{n} p_{j}\right)}{d \tau}\right]}{d\left(\prod_{j=5}^{n} p_{j}\right)} \cdot \frac{d\left(\prod_{j=5}^{n} p_{j}\right)}{d \tau}+\frac{C^{2}}{\prod_{j=5}^{n} p_{j}^{3}}=0
$$

in which by integration becomes

$$
\begin{aligned}
& \int \frac{d\left(\prod_{j=5}^{n} p_{j}\right)}{d \tau} \cdot d\left[\frac{d\left(\prod_{j=5}^{n} p_{j}\right)}{d \tau}\right]=\frac{1}{2}\left[\frac{d\left(\prod_{j=5}^{n} p_{j}\right)}{d \tau}\right]^{2}=\frac{1}{2}\left[\frac{d\left(\prod_{j=5}^{n} p_{j}\right)}{d t} \cdot \frac{d t}{d \tau}\right]^{2}= \\
& =\left[\frac{d\left(\prod_{j=5}^{n} p_{j}\right)}{d t}\right]^{2} \cdot \frac{C^{2}}{2 \prod_{j=5}^{n} p_{j}^{4}}=-C^{2} \cdot \int \frac{d\left(\prod_{j=5}^{n} p_{j}\right)}{\prod_{j=5}^{n} p_{j}^{3}}=\frac{C^{2}}{2 \prod_{j=5}^{n} p_{j}^{2}}+B=\frac{C^{2}+2 B \cdot \prod_{j=5}^{n} p_{j}^{2}}{2 \prod_{j=5}^{n} p_{j}^{2}}
\end{aligned}
$$


and

$$
\frac{d\left(\prod_{j=5}^{n} p_{j}\right)}{d t}= \pm \frac{\prod_{j=5}^{n} p_{j} \cdot \sqrt{C^{2}+2 B \cdot \prod_{j=5}^{n} p_{j}^{2}}}{C}= \pm \prod_{j=5}^{n} p_{j} \cdot \sqrt{1+\frac{2 B}{C^{2}} \cdot \prod_{j=5}^{n} p_{j}^{2}}
$$

By substituting (106) in (103), the 3D particles acceleration becomes

$$
\frac{d^{2} \sqrt{\sum_{i=1}^{4} X_{i}^{2}}}{d t^{2}}= \pm \frac{2 k}{C} \cdot \prod_{j=5}^{n} p_{j}^{2} \cdot \sqrt{1+\frac{2 B}{C^{2}} \cdot \prod_{j=5}^{n} p_{j}^{2}}= \pm 2 K \cdot \prod_{j=5}^{n} p_{j}^{2} \cdot \sqrt{1+\frac{2 B}{C^{2}} \cdot \prod_{j=5}^{n} p_{j}^{2}}
$$

where $B=$ const. . 